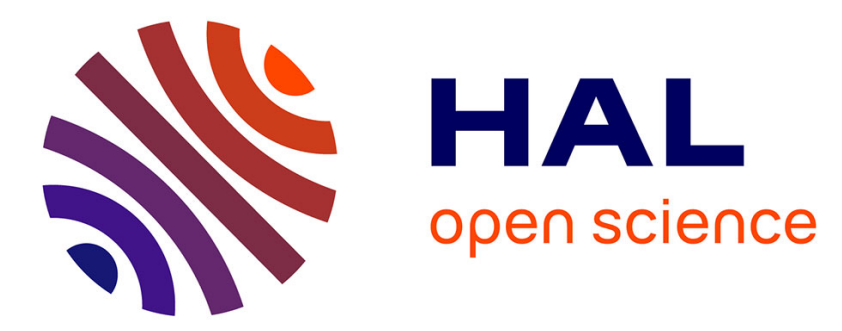

\title{
Riboswitches. Sequestration of a two-component response regulator by a riboswitch-regulated noncoding RNA.
}

J R Mellin, Mikael Koutero, Daniel Dar, Marie-Anne Nahori, Rotem Sorek, Pascale Cossart

\section{To cite this version:}

J R Mellin, Mikael Koutero, Daniel Dar, Marie-Anne Nahori, Rotem Sorek, et al.. Riboswitches. Sequestration of a two-component response regulator by a riboswitch-regulated noncoding RNA.. Science, 2014, 345 (6199), pp.940-3. 10.1126/science.1255083 . pasteur-01120664

\section{HAL Id: pasteur-01120664}

https://hal-pasteur.archives-ouvertes.fr/pasteur-01120664

Submitted on 24 Apr 2015

HAL is a multi-disciplinary open access archive for the deposit and dissemination of scientific research documents, whether they are published or not. The documents may come from teaching and research institutions in France or abroad, or from public or private research centers.
L'archive ouverte pluridisciplinaire HAL, est destinée au dépôt et à la diffusion de documents scientifiques de niveau recherche, publiés ou non, émanant des établissements d'enseignement et de recherche français ou étrangers, des laboratoires publics ou privés.

\section{(ㅇ)(1) $\$$}

Distributed under a Creative Commons Attribution - NonCommercial - NoDerivatives 44.0 


\section{Sequestration of a two-component response regulator by a riboswitch- regulated non-coding RNA}

J.R. Mellin ${ }^{12}$, Mikael Koutero ${ }^{12}$, Daniel Dar ${ }^{4}$, Marie-Anne Nahori ${ }^{12}$, Rotem Sorek $^{4}$, Pascale Cossart ${ }^{123^{*}}$

${ }^{1}$ Institut Pasteur, Unité des Interactions Bactéries-Cellules, Paris, F-75015 France. ${ }^{2}$ INSERM, U604, Paris, F-75015 France.

${ }^{3}$ INRA, USC2020, Paris, F-75015 France.

${ }^{4}$ Weizmann Institute of Science, Department of Molecular Genetics, Rehovot, Israel "Correspondence: pcossart@pasteur.fr 


\section{Abstract:}

Riboswitches are ligand-binding elements contained within the 5' untranslated

3 regions of bacterial transcripts, which generally regulate expression of downstream

4 open reading frames. Here we show that in Listeria monocytogenes, a Vitamin $\mathrm{B}_{12^{-}}$

5 binding $\left(B_{12}\right)$ riboswitch controls expression of a non-coding regulatory RNA, Rli55.

6 Rli55 in turn controls expression of the eut genes, which enable ethanolamine

7 utilization and require $B_{12}$ as a cofactor. Defects in ethanolamine utilization, or in its

8 regulation by Rli55, significantly attenuate Listeria virulence in mice. Rli55 functions

9 by sequestering the two-component response regulator, EutV via a EutV binding-site

10 contained within the RNA. Thus Rli55 is a riboswitch-regulated member of the small

11 group of regulatory RNAs that function by sequestering a protein and reveals a

12 unique mechanism of signal integration in bacterial gene regulation. 
Ethanolamine is an abundant molecule in the vertebrate intestine $(1,2)$, and

2 genes of the ethanolamine utilization pathway (eut) are widely conserved in

3 pathogenic bacteria (3-5). This includes the Gram positive intracellular human

4 pathogen Listeria monocytogenes, in which eut expression has been shown to be up-

5 regulated in the intestine during infection of mice (6), suggesting that ethanolamine is

6 important for Listeria pathogenesis. In Enterococcus faecalis eut expression is

7 activated in response to ethanolamine by a two-component response regulator,

8 EutVW $(7,8)$. Ethanolamine utilization requires $B_{12}$ as a cofactor $(9)$, and we noted

9 the presence of $a B_{12}$-binding riboswitch located upstream of the first gene in the eut

10 locus of L. monocytogenes (Fig. 1A) (10), suggesting eut expression might also be

11 regulated in response to $B_{12}$ availability.

12 To investigate a role for $\mathrm{B}_{12}$, we examined expression of the eut locus in 13 response to $B_{12}$ and ethanolamine by RNA-seq (Fig. 1B) and qRT-PCR (fig. S1). We 14 observed expression of the eutVW genes under all conditions, albeit at low levels, 15 suggesting that the cell maintains a pool of EutVW to sense and respond to 16 ethanolamine. In contrast, higher-level expression of eutVW and expression of other 17 eut genes requires both $B_{12}$ and ethanolamine (Fig. 1B). These data indicated $B_{12}$ is 18 required to activate eut expression and suggested that the $B_{12}$ riboswitch does not 19 prevent transcription of the eut locus in the presence of $B_{12}$, as might be expected for a classical riboswitch (11).

To clarify if the riboswitch has a role in the $B_{12}$-dependent regulation of eut expression we examined transcription of the riboswitch locus (Fig. 1C) in response to $\mathrm{B}_{12}$ and ethanolamine. We were unable to detect any long transcript, which might extend into the downstream eut locus. However, we did detect a $\sim 450$ nt transcript, Rli55 $(10,12)$ in the absence of $B_{12}$ (Fig. 1D), and a smaller ( 200nt) transcript which accumulated in the presence of $B_{12}$ (Fig. 1D). This result suggested the riboswitch 27 mediates transcription termination of the $\sim 450$ nt Rli55 transcript in response to $B_{12}$, 28 and any remaining long transcript is rapidly degraded (fig. S2A). A strain with a 
1 deletion in the $\mathrm{B}_{12}$ riboswitch ( $\Delta$ ribo), constitutively expressed the long Rli55

2 transcript under all conditions (Fig. 1E, fig. S2B), confirming $B_{12}$-dependent regulation

3 by the riboswitch. Conversely, neither the long or short Rli55 transcripts were 4 detected in a strain ( $\Delta$ rli55) in which the entire rli55 locus was deleted (Fig. 1E, fig. $5 \mathrm{~S} 2 \mathrm{~B})$. Thus the $\mathrm{B}_{12}$ riboswitch determines whether Rli55 is expressed as a long or 6 short transcript in response to $B_{12}$.

We reasoned Rli55 might act as a regulatory RNA controlling expression of

8 the eut locus. To test this hypothesis we examined expression of eut genes by qRT-

9 PCR in the wild-type, $\Delta$ ribo and $\Delta$ rli55 strains. In the wild-type strain eut expression 10 was elevated only when both ethanolamine and $B_{12}$ were present in the media (Fig $111 \mathrm{~F}$, fig S3A\&B), whereas in the $\Delta$ ribo strain, which constitutively expresses the long 12 Rli55, eut genes were never expressed under any conditions. In contrast, in the $13 \Delta$ rli55 strain, expression of the eut genes was high in the presence of ethanolamine 14 alone in addition to ethanolamine and $B_{12}$ together. Together, these data support a impacted L. monocytogenes virulence, by examining the wild-type, $\Delta$ ribo and $\Delta r l i 55$ in comparable amounts to the wild-type strain in the spleen and liver. Thus defects in 27 ethanolamine utilization or activation of eut expression significantly attenuate $L$. monocytogenes virulence. 
In E. faecalis, ethanolamine is sensed by the sensor-kinase EutW, which

2 subsequently phosphorylates the response regulator, EutV (7). Phosphorylated EutV

3 in turn binds ANTAR elements (AmiR and NasR Transcriptional Antiterminator

4 Regulator) in the 5'UTRs of actively transcribed eut mRNAs, which prevents the

5 formation of a transcription terminator and consequently activates eut expression

6 (13). In L. monocytogenes ANTAR sites were identified upstream of the eutA and

7 eutV genes and a third site was identified in the rli55 locus upstream of the eutG

8 gene and downstream of the $B_{12}$ riboswitch (13). We also identified a second ANTAR

9 site in the rli55 locus (Fig. 3A), suggesting that one or both of these ANTAR elements

10 could be transcribed as part of the 3' end of Rli55 RNA. Indeed, our RNA-seq data

11 showed that, in the absence of $B_{12}$, high levels of Rli55 are transcribed as a long 12 transcript encompassing the first ANTAR element (Fig. 3A). In contrast, rli55

13 transcription terminates abruptly after the riboswitch in the presence of $B_{12}$, indicating

14 the riboswitch determines if Rli55 is transcribed with or without an ANTAR element.

15 This notion was supported by the detection of putative Rli55 orthologues in $E$.

16 faecalis and Streptococcus sanguinis (fig. S4). In the latter, the riboswitch, in 17 conjunction with a single ANTAR element, has undergone an inversion relative to the 18 adjacent eutG gene, suggesting the riboswitch and the first ANTAR element are 19 functionally linked (fig. S4B), and that this is also the case in L. monocytogenes.

To test if the ANTAR elements are involved in Rli55-mediated regulation, we 21 complemented the $\Delta$ rli55 strain with chromosomally integrated rli55 alleles carrying 22 mutations in the ANTAR elements and examined which ones restored Rli5523 mediated regulation of eut expression (Fig 3B\&C, fig. S5). A strain with an empty 24 construct ( $\Delta$ rli55::Empty) could not prevent expression of the eut genes in 25 ethanolamine alone (as in the parental $\Delta$ rli55 strain), whereas a strain with a wildtype copy of rli55 ( $\Delta$ rli55::rli55) fully restored Rli55-mediated inhibition. However, a

27 strain with a deletion in the riboswitch ( $\Delta$ rli55:: $\Delta$ ribo) inhibited eut expression in all 28 conditions, as the riboswitch can no longer terminate rli55 transcription in response to 
$1 \quad \mathrm{~B}_{12}$. In a strain $\Delta r l i 55::$ rli55 $\Delta \mathrm{M} 1$, wherein four uridine residues in the first ANTAR site

2 were mutated to adenines (Fig. 3D, $\Delta M 1$ ), inhibition of eut expression by Rli55 was

3 abolished in the presence of ethanolamine alone. In contrast, in strain

$4 \Delta$ sli55::rli55 $\Delta \mathrm{M} 1 / \mathrm{M} 2$, where compensatory mutations were made to the opposite side

5 of the ANTAR stem-loop (Fig. 3D, $\Delta M 2$ ), wild-type regulation of eut expression was

6 restored. Mutation of the six nucleotides in the stem-loop of the second ANTAR

7 element (Fig. 3D, $\Delta \mathrm{M} 3, \Delta r$ rli55::rli55 $\Delta \mathrm{M} 3$ ) had no significant effect on Rli55-mediated

8 regulation. Thus the first ANTAR element is necessary and sufficient for Rli55-

9 mediated regulation.

The long form of Rli55 containing an ANTAR element might bind and sequester EutV, preventing it from activating expression of the eut genes in the presence of ethanolamine but absence of $B_{12}$. When sufficient levels of $B_{12}$ accumulate, $\mathrm{B}_{12}$ would bind the riboswitch, producing truncated Rli55 transcripts, which would lack an ANTAR element and be unable to sequester EutV. To examine this hypothesis, we constructed a strain with an additional copy of the eutV gene carrying a 2XFLAG-tag (EutV ${ }^{\text {FLAG }}$ ) and first showed that expression of EutV ${ }^{\text {FLAG }}$ protein is regulated identically to the native eutV gene in response to ethanolamine and $\mathrm{B}_{12}$ (fig. S6A). We also constructed a strain with an additional eutV gene lacking a FLAG tag (EutV NOFLAG). Anti-FLAG immunoprecipitations with cell lysates from these two strains (fig. S6B\&C) followed by RNA-seq analysis (Fig. 3E\&F) showed that Rli55 is enriched by co-immunoprecipitation with EutV ${ }^{\text {FLAG }}$ primarily when bacteria are grown in the presence of ethanolamine alone, while we saw no enrichment in a parallel immunoprecipitation with the EutV ${ }^{\text {NOFLAG }}$ strain (Fig. 3E). In contrast, the ANTAR element upstream of the eutV gene (Fig. 3F) is enriched by coimmunoprecipitation of lysates from EutV $V^{F L A G}$ bacteria, but not EutV ${ }^{\text {NOFLAG }}$ bacteria,

26 grown in the presence of ethanolamine and $B_{12}$ together but not from lysates of 27 bacteria grown in ethanolamine alone. To a lesser extent the ANTAR containing 28 region upstream of eutA and the entire eutA-Q locus is enriched under the latter 
1 condition (fig. S7). These data support a model in which the majority of EutV is bound

2 and sequestered by Rli55 in the presence of ethanolamine alone. Conversely, in the

3 presence of ethanolamine and $B_{12}$, the riboswitch produces short truncated Rli55

4 transcripts, which cannot bind EutV, allowing EutV to bind eut mRNAs and activate

5 eut expression (Fig. 3G).

6 This riboregulatory mechanism coordinates expression of the ethanolamine 7 utilization (eut) locus with the availability of $B_{12}$, the essential cofactor for 8 ethanolamine catabolism. Our results highlight a so-far undescribed role for 9 ethanolamine utilization in infection. Previously ethanolamine utilization has been 10 shown to be important after oral infection $(3,4,14)$, however, the contribution of 11 ethanolamine utilization to L. monocytogenes pathogenesis in an intravenous mouse 12 infection model suggests that ethanolamine utilization is important outside of the 13 intestine and possibly in the intracellular environment. This study also extends the 14 role of riboswitches in the regulation of non-coding RNAs $(15,16)$. Finally, our data 15 show that Rli55 represents a new member of the small family of regulatory RNAs that 16 function by sequestering a protein, which also includes the $6 S$ and CsrB/C RNAs (17) 17 and highlights a unique means of signal integration in bacterial gene regulation. 
1 Figure 1. Control of Ethanolamine utilization (eut) genes by a $B_{12}$-riboswitch

2 regulated ncRNA. (A) The eut locus. Green arrow denotes $B_{12}$ riboswitch. Vertical

3 arrows denote positions of ANTAR elements (13) (B) RNA-seq coverage of eut

4 operon. (C) Regions deleted in the $\Delta r l i 55$ and $\Delta$ ribo strains are indicated by solid

5 black lines. Northern blot of the rli55 transcript in the (D) wild-type strain with probe

6 indicated by thick dashed line in schematic or (E) wild-type (wt), $\Delta$ ribo or $\Delta$ rli55

7 strains with probe indicated by thin dashed line in schematic. Ethidium bromide

8 staining of rRNA is shown as a control. Quantitation of bands is shown below each

9 blot. Expression of the (F) eutV, eutA \& eutT genes was evaluated by qRT-PCR in 10 the indicated strains. Values represent means $+/-$ SEM, $n=3,{ }^{*}=p<0.05$, ns $=$ not 11 significant.

Figure 2. Ethanolamine utilization and pathogenesis. BalbC mice were injected intravenously with $\sim 4,500 \mathrm{CFU}$ of the indicated strain. Mice were sacrificed at (A \& B) $24 \mathrm{hr}$, (C \& D) $48 \mathrm{hr}$ or (E \& F) $72 \mathrm{hr}$ and spleens and livers removed to assess bacterial load per organ. Results represent two independent experiments with 3-4 mice per group in each experiment. ${ }^{*}=p<0.05$, ns $=$ not significant.

Figure 3. Rli55 sequesters the two-component response regulator EutV. (A) RNA-seq coverage in reads per million (RPM) of the rli55 locus from bacteria grown in as indicated. (B \& C) qRT-PCR of eutV \& eutA genes. vitamin $B_{12}\left(B_{12}\right)$, ethanolamine (Ea). Values represent means + /- SEM, $n=3$. All differences were significantly different $\left({ }^{*}=p<0.05\right)$ from the $\Delta$ rli55::Empty strain in the Ea condition, except where indicated (ns). All differences were not significantly different from the $\Delta r l i 55::$ Empty strain in the $\mathrm{B}_{12}+$ Ea condition, except where indicated (D) Secondary

26 structure of the ANTAR elements encoded in the boxed region of Fig. 3A. Mutations 27 are shown in orange. (E \& F) Histogram shows RNA-Seq coverage in reads/million 28 (RPM) of the rli55 and eutV loci with RNA isolated by co-immunoprecipitation of cell 
1 lysates from either EutV ${ }^{F L A G}$ or EutV ${ }^{\text {NOFLAG }}$ cultures grown in the presence of 2 ethanolamine (Ea) or ethanolamine and $B_{12}$ together. (G) Proposed model of Rli553 mediated regulation of EutV in the presence of ethanolamine (Ea) alone or 4 ethanolamine $+B_{12}$ 


\section{References:}

1. D. A. Garsin, Ethanolamine utilization in bacterial pathogens: roles and regulation. Nat. Rev. Microbiol. 8, 290-295 (2010).

2. K. Kawai, M. Fujita, M. Nakao, Lipid components of two different regions of an intestinal epithelial cell membrane of mouse. Biochim. Biophys. Acta 369, 222-233 (1974).

3. P. Thiennimitr et al., Intestinal inflammation allows Salmonella to use ethanolamine to compete with the microbiota. Proc Natl Acad Sci USA 108, 17480-17485 (2011).

4. Y. Bertin et al., Enterohaemorrhagic Escherichia coli gains a competitive advantage by using ethanolamine as a nitrogen source in the bovine intestinal content. Environ. Microbiol. 13, 365-377 (2011).

5. O. Tsoy, D. Ravcheev, A. Mushegian, Comparative genomics of ethanolamine utilization. J Bacteriol 191, 7157-7164 (2009).

6. C. Archambaud et al., Impact of lactobacilli on orally acquired listeriosis. Proc Natl Acad Sci USA 109, 16684-16689 (2012).

7. M. F. Del Papa, M. Perego, Ethanolamine activates a sensor histidine kinase regulating its utilization in Enterococcus faecalis. J Bacteriol 190, 7147-7156 (2008).

8. K. A. Fox et al., Multiple posttranscriptional regulatory mechanisms partner to control ethanolamine utilization in Enterococcus faecalis. Proc Natl Acad Sci USA 106, 4435-4440 (2009).

9. J. R. Roth, J. G. Lawrence, T. A. Bobik, Cobalamin (coenzyme B12): synthesis and biological significance. Annu Rev Microbiol 50, 137-181 (1996).

10. A. Toledo-Arana et al., The Listeria transcriptional landscape from saprophytism to virulence. Nature 459, 950-956 (2009).

11. W. C. Winkler, R. R. Breaker, Regulation of bacterial gene expression by riboswitches. Annu Rev Microbiol 59, 487-517 (2005).

12. O. Wurtzel et al., Comparative transcriptomics of pathogenic and nonpathogenic Listeria species. Mol. Syst. Biol. 8, 583 (2012).

13. A. Ramesh et al., The mechanism for RNA recognition by ANTAR regulators of gene expression. PLoS Genet. 8, e1002666 (2012).

14. S. E. Winter, A. J. Bäumler, A breathtaking feat: to compete with the gut microbiota, Salmonella drives its host to provide a respiratory electron acceptor. Gut Microbes 2, 58-60 (2011).

15. J. R. Mellin et al., A riboswitch-regulated antisense RNA in Listeria monocytogenes. Proc Natl Acad Sci USA 110, 13132-13137 (2013).

16. G. André et al., S-box and T-box riboswitches and antisense RNA control a sulfur metabolic operon of Clostridium acetobutylicum. Nucleic Acids Res 36, 5955-5969 (2008). 
1 17. S. Gottesman, G. Storz, Bacterial Small RNA Regulators: Versatile Roles and Rapidly Evolving Variations. Cold Spring Harb Perspect Biol (2010), doi:10.1101/cshperspect.a003798.

4

\section{Acknowledgments:}

6 We are grateful to Wade Winkler, Danielle Garsin, Nicole King and Joseph Pederson

7 for helpful and insightful discussions, and Yanjie Chao and Jörg Vogel for assistance 8 with the CLIP-Seq protocol. This work was supported by grants to PC, ERC 9 Advanced grant (233348), ANR (BACNET 09-BLAN-0024-02), ANR Investissement 10 d'Avenir Programme (10-LABX-62), Fondation Le Roch and Fondation Jeantet. PC is 11 a International Senior Research Scholar of the Howard Hughes Medical Institute.

13 Supplementary Materials:

14 Materials and Methods

15 Figures S1-S7

16 Tables S1-S2 
A ethanolamine utilization (eut) operon

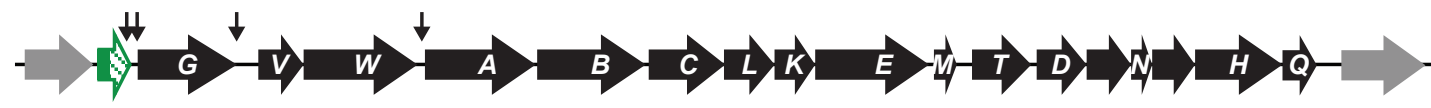

B

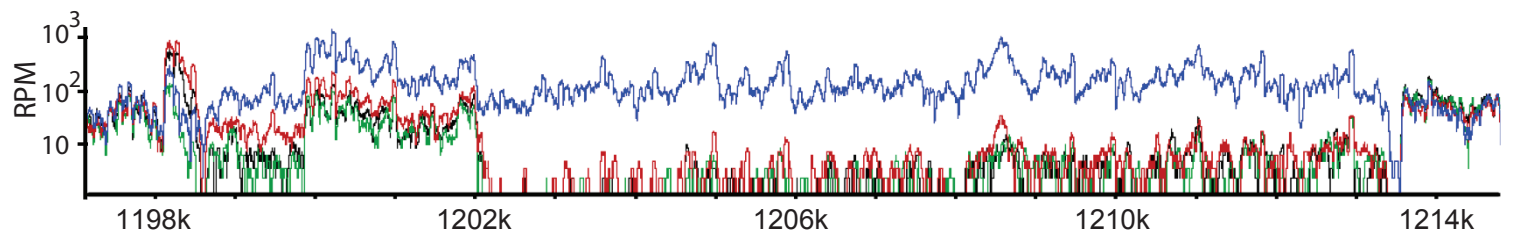

- LB

- B12

- Ea

- B12+Ea

C

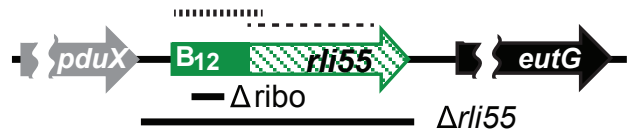

F

E

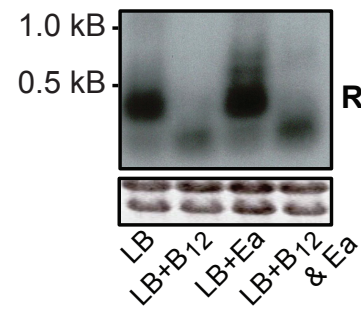

흥 $\stackrel{\oplus}{=} 1.5$

丩 তั 0.5 .

long rli55

丩

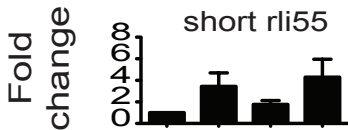

Rli55
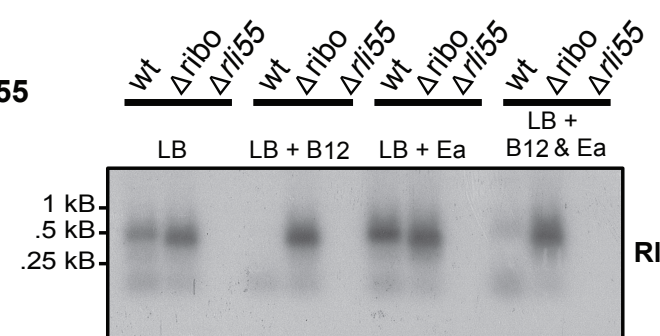

Rli55

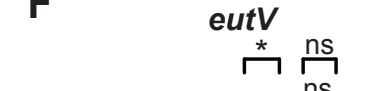

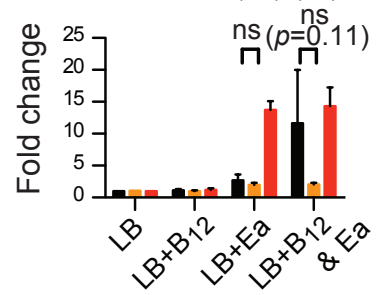

eutC

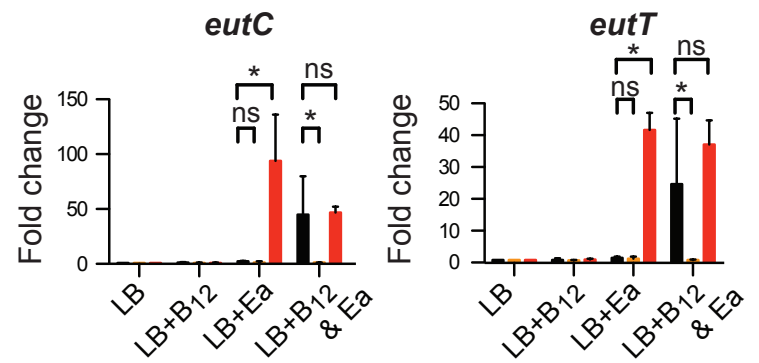


A

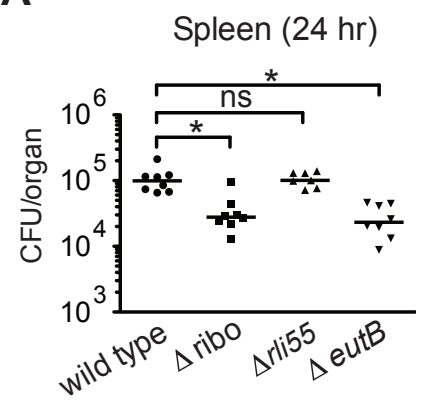

C

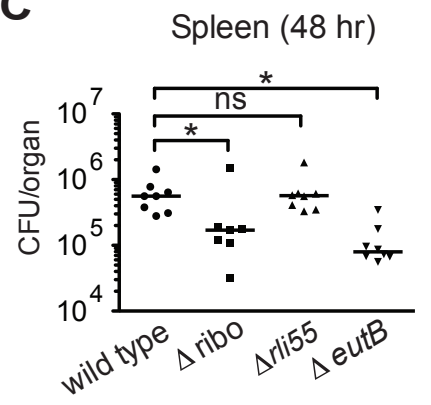

E Spleen (72 hr)

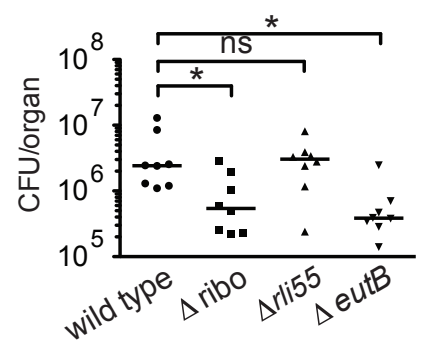

B

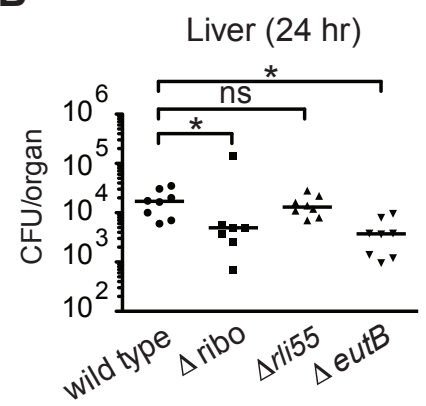

D

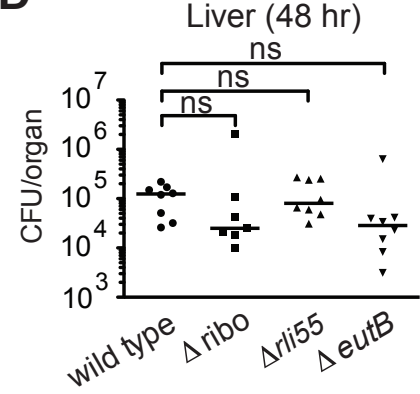

F

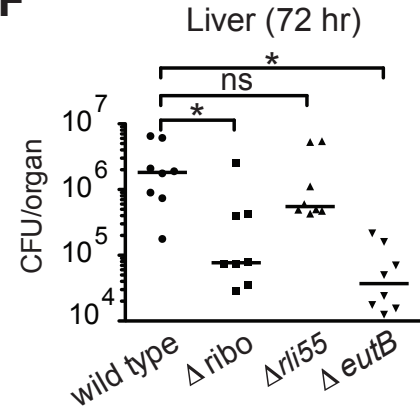


A

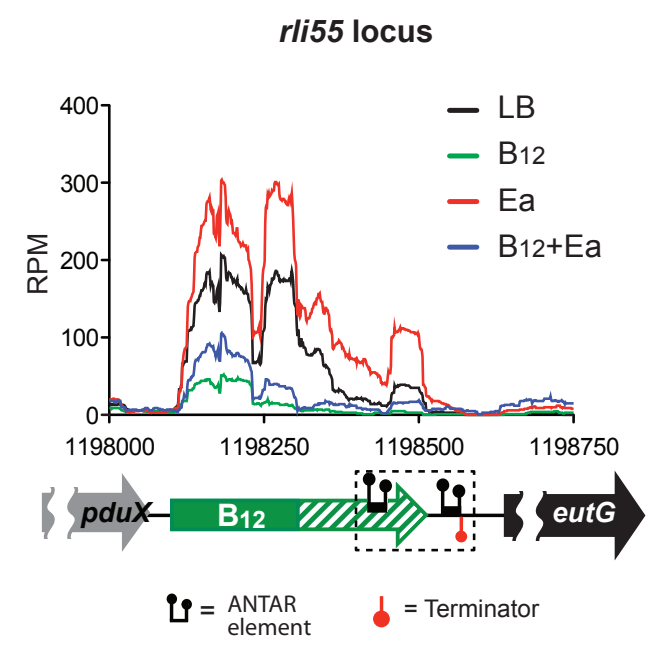

E

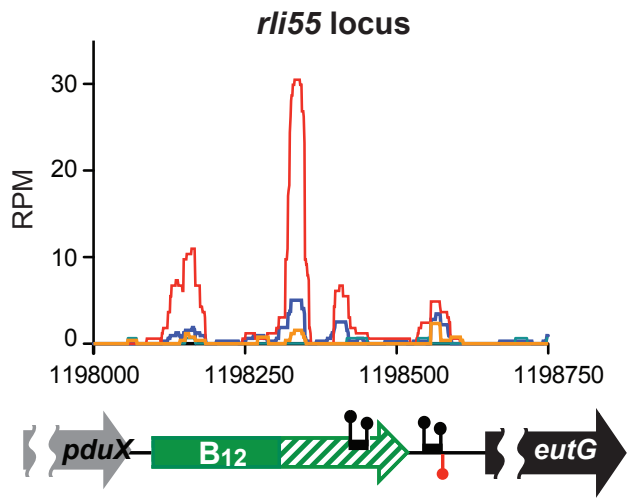

F
B
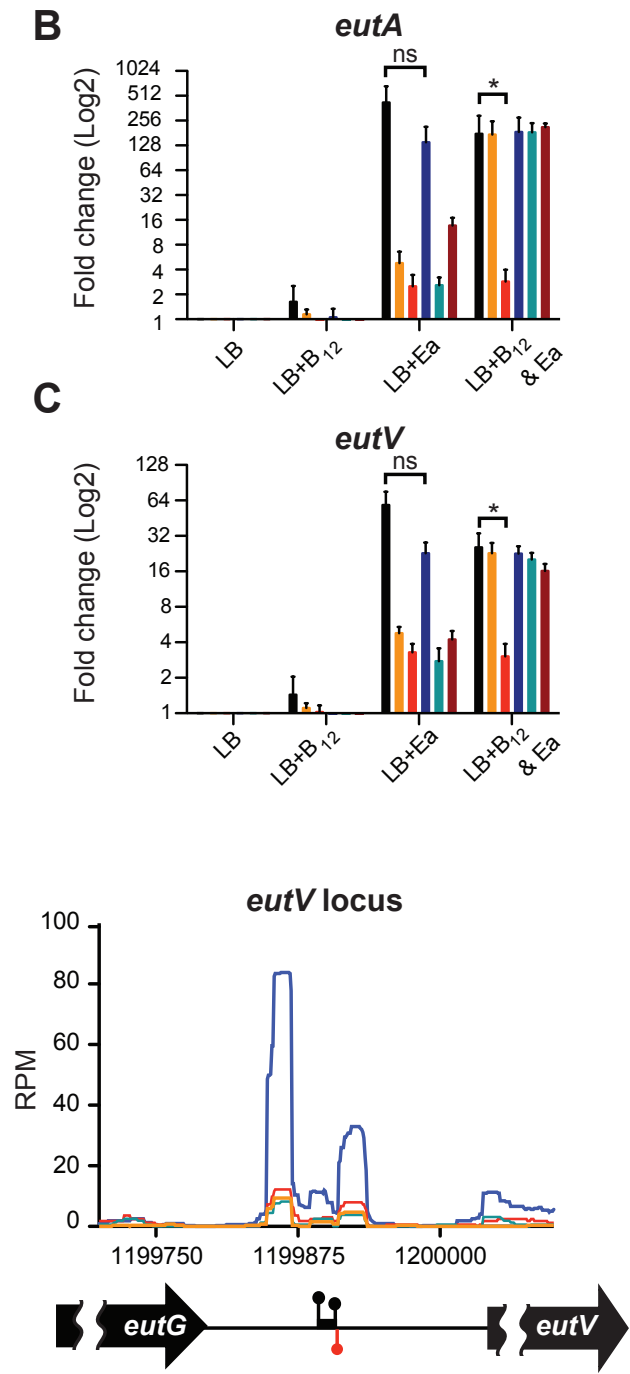

D
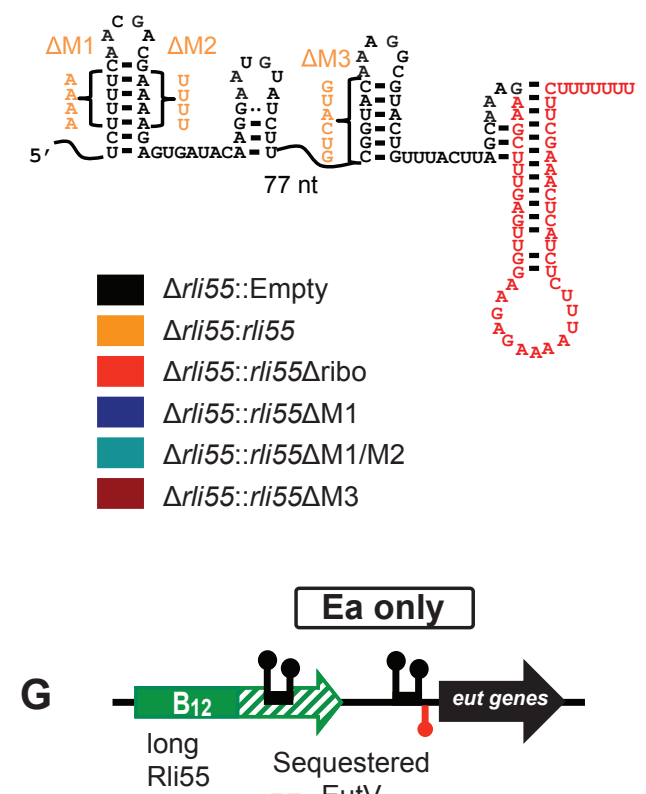
EutV
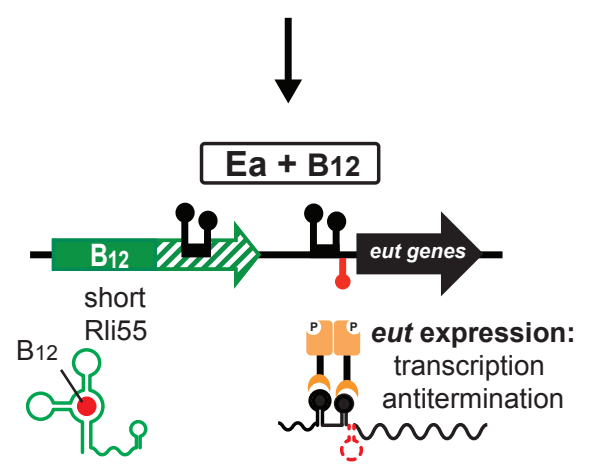


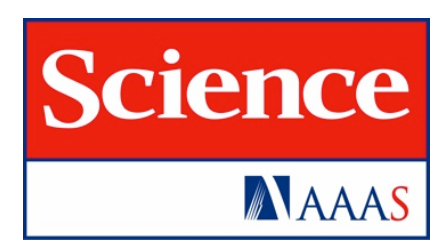

Supplementary Materials for

Sequestration of a two-component response regulator by a riboswitchregulated non-coding RNA

\author{
J.R. Mellin ${ }^{123}$, Mikael Koutero ${ }^{123}$, Daniel Dar ${ }^{4}$, Marie-Anne Nahori $^{123}$, \\ Rotem Sorek ${ }^{4} \&$ Pascale Cossart ${ }^{123^{*}}$ \\ ${ }^{1}$ Institut Pasteur, Unité des Interactions Bactéries-Cellules, Paris, F-75015 \\ France. \\ ${ }^{2}$ INSERM, U604, Paris, F-75015 France. \\ ${ }^{3}$ INRA, USC2020, Paris, F-75015 France. \\ ${ }^{4}$ Weizmann Institute of Science, Department of Molecular Genetics, Rehovot, \\ Israel \\ correspondence to: pcossart@pasteur.fr
}

This PDF file includes:

Materials and Methods

Figs. S1 to S7

Tables S1 to S2 


\section{Materials and Methods}

\section{Bacterial Strains, Plasmids, Primers and Growth Conditions}

Bacterial strains and plasmids used in this study are listed in Table S1. Primers used in this study are listed in Table S2. For all northern blot and qRTPCR experiments Listeria monocytogenes and Enterococcus faecalis were grown in LB broth with shaking at $200 \mathrm{rpm}$ in Erlenmeyer flasks at $37^{\circ} \mathrm{C}$. Streptococcus sanguinis was grown in $\mathrm{BHI}$ broth. $\mathrm{LB}$ and $\mathrm{BHI}$ were supplemented with $10 \mathrm{mM}$ ethanolamine (Sigma, 98136) or $20 \mathrm{~nm}$ Vitamin $\mathrm{B}_{12}$ (Sigma. C0884), as indicated. E. coli was grown in LB broth. For all RNA isolations bacteria were inoculated to $\mathrm{OD}_{600} 0.02$ and grown to and $\mathrm{OD}_{600}$ of 0.3 -0.5 .

\section{Constructions of Plasmids and Strains}

All cloning followed standard molecular biology protocols. For construction of pMAD-based plasmids for generation of mutants, regions of $500-700 \mathrm{nt}$ regions of complementarity both upstream and downstream of a targeted region were PCR amplified with Phusion High fidelity polymerase and reagents (Finnzymes, F-553) using genomic DNA as a template. Upstream and downstream regions were joined with a second overlapping extension PCR reaction using the first two PCR products as template to generate a "UD" PCR product. UD PCR products were subsequently purified with QIAquick PCR purification columns (Qiagen, 28104), digested with the appropriate restriction enzyme (New England Biolabs), purified again as before, and ligated into digested pMAD plasmid at overnight at $16^{\circ} \mathrm{C}$ with T4 DNA ligase (New England Biolabs, M0202S). $2 \mu \mathrm{l}$ of each ligation were transformed into chemically competent E. coli Top10 (Invitrogen, C404003) cells according to the manufacturers instructions. Transformants were screened by PCR for the presence of the appropriate insert and purified plasmid was isolated from positive colonies by miniprep using QIAprep spin columns (Qiagen, 27104). Electrocompetent $L$. monocytogenes strains were transformed with each respective plasmid and grown at $30^{\circ} \mathrm{C}$ for two days on $\mathrm{BHI} \mathrm{X}-\mathrm{Gal}$ plates. Blue colonies were selected and grown in $\mathrm{BHI}$ at $30^{\circ} \mathrm{C}$ for 6 hours. Cultures were subsequently diluted $1: 1000$ in fresh $\mathrm{BHI}$ and grown overnight at $42^{\circ} \mathrm{C}$. Culture were serially diluted and plated onto BHI X-Gal (5-bromo-4-chloro-3-indolyl- $\beta$-Dgalactopyranoside) $(80 \mu \mathrm{g} / \mathrm{ml})$, Erythromycin $(5 \mu \mathrm{g} / \mathrm{ml})$ plates. Blue, resistant colonies have the plasmid integrated into the chromosome, as the pMAD plasmid cannot replicate at high temperature. Colonies with the plasmid integrated into the chromosome were subsequently inoculated into fresh $\mathrm{BHI}$ and grown at $30^{\circ}$ $\mathrm{C}$ the subsequent day for $6 \mathrm{hrs}$ and then shifted to $42^{\circ} \mathrm{C}$ for three hours. Cultures were again serially diluted and plated onto BHI, X-Gal plates without selection. White colonies were screened by PCR, for loss of the plasmid, which can occur through recombination with the repeated upstream or downstream regions, to either regenerate the wild-type chromosome or a deletion mutant. All mutants were confirmed by DNA sequencing. For complementation plasmids, 
genomic DNA was used to amplify a fragment containing the rli55 gene. Subsequent mutations were introduced by site-directed mutagenesis using primers containing mismatches at the desired bases. Briefly, two fragments containing overlapping regions with the desired mutation were produced and then joined by a subsequent splice overlap extension PCR, digestion, and ligation into the pJM2 vector. For the construction of the EutV ${ }^{F L A G}$ strain, a synthetic gene containing a C-terminal FLAG tag was ordered from IDT DNA, and used as a template for amplification and subsequent digestion and cloning into the pJM2 vector.

\section{RNA isolation}

For all RNA isolations, $25 \mathrm{ml}$ cultures of L. monocytogenes were grown to an $\mathrm{OD}_{600}$ of $0.3-0.5$ and subsequently pelleted by centrifugation at $4000 \mathrm{rpm}$ for 20 mins in $50 \mathrm{ml}$ Falcon tubes with swing bucket rotor. Pellets were resuspended in $1 \mathrm{ml}$ Tri Reagent (Sigma, 93289), transferred to $2 \mathrm{ml}$ Lysing Matrix Tubes (MP Biomedicals, 6911) and mechanically lysed by bead beating in a FastPrep apparatus ( 45 secs, speed 6.5 followed by an additional 30 secs, speed 6.5). Subsequently tubes were centrifuged for 5 min at $8000 \times$ G, $4^{\circ} \mathrm{C}$ in a tabletop centrifuge to separate beads from lysates. Lysates were drawn off and transferred to $2 \mathrm{ml}$ Eppendorf tubes. RNA isolation proceeded according to the manufacturers instructions. Briefly, $200 \mu \mathrm{l}$ Chloroform (Carlo Erba Reagents, 438601) was added to the lysate, shaken and incubated for 10 minutes at room temperature followed by centrifugation for $15 \mathrm{~min}$ at $13,000 \times \mathrm{G}, 4^{\circ} \mathrm{C}$. The upper aqueous phase was removed and transferred to a new $1.5 \mathrm{ml}$ Eppendorf tube and RNA was precipitated by the addition of $500 \mu \mathrm{l}$ Isopropanol and incubation at room temperature for 5-10 mins. RNA was pelleted by centrifuging for 10 mins at $13,000 \times \mathrm{G}, 4^{\circ} \mathrm{C}$. The supernatant was discarded and the pellet was washed twice with $75 \%$ ethanol. RNA pellets were resuspended in $50 \mu \mathrm{l}$ water.

\section{Northern Blots}

RNA probes radioactively labeled with $\alpha$-32P-UTP (Perkin-Elmer, BLU007X500UC) were generated using the T7 Maxiscript kit (Ambion, AM1314) and PCR templates were amplified from genomic DNA using primers listed in Table S2. For each sample, $10-20 \mu \mathrm{g}$ of RNA was incubated 1:2 by volume with Formaldehyde loading dye (Ambion, 8552) supplemented with $20 \mu \mathrm{g} / \mathrm{ml}$ ethidium bromide, and incubated at $65^{\circ} \mathrm{C}$ for 15 min to denature RNA secondary structures. Samples were loaded onto $1 \%$ agarose gels (Ambion, 9040) and electrophoresed in Northernmax Denaturing Gel Buffer (Ambion, AM8676) at 60 $\mathrm{mV}$ for approximately $2 \mathrm{hrs}$. RNA was transferred to Nytran membranes (Sigma, Z670286) by passive downward transfer with 20X SSC. Membranes were photographed under ultraviolet light to capture ethidium bromide staining of ribosomal RNA bands for loading controls. The positions of RNA size markers (Ambion, AM7150 or a in-house generated set) were marked with a scalpel. RNA was crosslinked to membranes by exposure to UV light for five minutes and membranes were prehybridized in Ultrahyb buffer (Ambion, AM8670) for 30 mins 
before probes were added an incubated overnight. Hybridizations were carried out at $60-68^{\circ} \mathrm{C}$. The following morning, membranes were washed twice for 5 mins in 2X SSC, $0.1 \%$ SDS at room temperature with constant agitation, followed by two washes in in $0.1 \mathrm{X}$ SSC, $0.1 \%$ SDS at the respective hybridization temperature with constant agitation. Membranes were wrapped in cellophane and subsequently used to expose films (GE Healthcare, 28906844). Quantitation of blots represent mean values of three biological replicate experiments +/- SD, normalized to loading controls.

\section{Determination of RNA half-life}

Strains were grown to an $\mathrm{OD}_{600}$ of $0.3-0.5$ in either ethanolamine alone $(0.01 \mathrm{mM})$ or ethanolamine $(0.01 \mathrm{mM})$ and $B_{12}(20 \mathrm{nM})$. Cells were subsequently treated with rifampicin $(500 \mu \mathrm{g} / \mathrm{ml}$ final concentration) and Nalidixic acid (40 $\mu \mathrm{g} / \mathrm{ml}$ final concentration), dissolved in DMSO, to halt further transcription and DNA synthesis. Aliquots were removed time 0 (just before treatment) or after treatment at $2 \mathrm{~min}, 5 \mathrm{~min}, 9 \mathrm{~min}, 16 \mathrm{~min}, 24 \mathrm{~min}$ and $33 \mathrm{~min}$. Cells were harvested by centrifugation in a tabletop centrifuge at 13,000 rpm for $1 \mathrm{~min}$. Pellets were resuspended in $1 \mathrm{ml}$ Trizol, flash frozen in liquid nitrogen and subsequently RNA was isolated as described above. For northern blots, $5 \mu \mathrm{g}$ of Total RNA was run on $1 \%$ agarose gels, and northerns were carried out as described above with either the T7riboprobe or the T716S RNA probes made by T7 RNA transcription as described above. Blots were imaged with a Typhoon FLA9000 phosphoimager and quantified with the MulitGauge V3.0 software suite. Values represent mean values of three biological replicate experiments $+/-\mathrm{SD}$, normalized to $16 \mathrm{~S}$ loading controls.

\section{Quantitative Real Time PCR}

For each sample, $10 \mu \mathrm{g}$ of RNA was treated with Turbo DNase (Ambion, AM2238) for $1 \mathrm{hr}$ at $37 \mathrm{C}$ in a $20 \mu \mathrm{l}$ reaction. RNA was subsequently purified by ammonium acetate precipitation, washed once with $75 \%$ ethanol and resuspended in water. cDNA was synthesized from $1 \mu \mathrm{g}$ of RNA using the iScript cDNA Synthesis kit (Bio-Rad, 170-8890) according to the manufacturer's instructions and reactions were subsequently diluted with $180 \mu \mathrm{l}$ of water. qRTPCR reactions were prepared in $10 \mu \mathrm{l}$ volumes on 384 well plates $(\mathrm{ABI}$, 4309849). Reactions were prepared as follows: $2 \mu$ l of diluted CDNA, $2 \mu \mathrm{l}$ water, $5 \mu \mathrm{l}$ SYBR Green master mix (ABI, 4309155), .5 $\mu \mathrm{M}$ of gene specific oligonucleotides, water to $10 \mu \mathrm{l}$ total volume. Oligonucleotides were designed using a primer design program available at http://eu.idtdna.com/Scitools/ Applications/Primerquest/, and are listed in Table S2. Primer efficiencies were evaluated by generating a standard curve with serial dilutions and shown to be between $90 \%-110 \%$ in all cases. Reactions were carried out and quantified on an $\mathrm{ABI}$ Prism $7900 \mathrm{HT}$ machine with SDS2.4 software. PCR conditions were as follows: $95^{\circ} \mathrm{C}$ for $10 \mathrm{~min}$ followed by 40 cycles of $95^{\circ} \mathrm{C} 15$ secs and $60^{\circ} \mathrm{C} 15$ secs. Specificity of the amplified product and primer dimer formation was verified for each primer set by the presence of only a single peak in a disassociation step 
carried out after each run. The absence of contaminating DNA was verified with control samples for each RNA sample for which no prior reverse transcription reaction was carried out. Expression levels of all genes were normalized to the $\mathrm{L}$. monocytogenes rpoB gene, and fold changes were calculated using the $\triangle \triangle C T$ method. All samples were evaluated in triplicate and at least three independent experiments were carried out for each gene evaluated. For statistical evaluation, all values were log2 converted and evaluated by paired t-test. Differences were considered statistically significant when $p$ values were $<0.05$.

\section{Intravenous mouse infections}

BalbC mice were injected intravenously with $5000 \mathrm{CFU}+/-1800 \mathrm{CFU}$ of the indicated strain. Inoculums were verified by plating of serially diluted aliquots onto $\mathrm{BHI}$ plates before and after injection followed by counting of colony forming units after overnight growth at $37^{\circ} \mathrm{C}$. Mice were sacrificed at subsequent timepoints and spleens and livers removed, homogenized and serially diluted. Dilutions were plated onto $\mathrm{BHI}$ plates and grown overnight at $37^{\circ} \mathrm{C}$. Colonies were counted to assess bacterial load per organ. Two independent experiments were carried out with 4 mice per group in each experiment. (Note: two data points were removed due to contamination of the plates, and one mice injected with the $\Delta$ ribo strain died within $24 \mathrm{hr}$ after injection, likely due to a misplaced injection, and was not included in the analysis). Statistically significant differences were evaluated by the Mann-Whitney test, and differences were considered statistically significant when $p$ values were $<0.05$.

\section{Whole transcriptome RNA-Sequencing}

$10 \mu \mathrm{g}$ DNase I treated RNA was depleted of ribosomal RNA using MicrobeExpress (Life Technologies, AM1905). $1 \mu \mathrm{g}$ RNA was fragmented at $72^{\circ} \mathrm{C}$ for 2 min using a fragmentation kit (Ambion, AM8740) and cleaned using AMPure XP magnetic beads (Agencourt, A63880). CDNA was generated by reverse transcription (RT) with random hexamers (Thermo Scientific, SO142) using the Affinity Script enzyme (Agilent, 930107-51) according to the manufacturer's instructions at a final volume of $20 \mu \mathrm{l}$. Second strand synthesis was performed in by adding the following to the completed RT reaction: $8 \mu \mathrm{l}$ NEB2 10X buffer, $3 \mu \mathrm{l}$ dNTPs $10 \mathrm{mM}, 3 \mu \mathrm{l}$ dATP $10 \mathrm{mM}, 24 \mathrm{U}$ of E. coli DNA polymerase I, $200 \mathrm{U}$ RNase $\mathrm{H}$ and $400 \mathrm{U}$ of T4 DNA ligase (New England BioLabs, M0202S). Final volume was adjusted to $80 \mu \mathrm{l}$ using DNase free water and the reaction was incubated at $16^{\circ} \mathrm{C}$ for $2 \mathrm{~h}$. dscDNA was cleaned using magnetic beads and then subjected to A-base addition and adapter ligation with adapters carrying the Illumina sequences as described (18). The resulting library was amplified with 16 cycles of PCR using the Kappa-HiFi enzyme (KAPA Biosystems, KK2601). Libraries were sequenced on the Hiseq2500 and reads were mapped to the corresponding reference genome (NC_003210) using Novoalign (Novocraft V3.02.02). Data analysis was performed using in-house Perl scripts. 


\section{CLIP-Seq (Cross-linking followed by imunnoprecipitation and RNA-Seq)}

I. Immunoprecipitation

The CLIP-seq protocol was adapted from (19). Strains carrying either a FLAG-tagged or untagged copy of the eutV gene in the $\operatorname{tRNA}^{\mathrm{ARG}}$ locus were cultured overnight in LB media. Overnight cultures were inoculated into $500 \mathrm{ml}$ of fresh LB media in 2L Erlenmeyer shake flasks to an $\mathrm{OD}_{600}$ of 0.02 and grown to $\mathrm{OD}_{600}$ 0.3-0.5. Cultures were centrifuged in a Sorval ultracentrifuge (RC26 Plus) at $8000 \mathrm{rpm}$ for 20 mins, resuspended in $20 \mathrm{ml}$ of fresh media, spread in 12 $\mathrm{cm} \mathrm{X} 12 \mathrm{~cm}$ dished to a thickness of $\sim 2 \mathrm{~mm}$ and exposed to $0.8 \mathrm{~J}$ of $254 \mathrm{~nm} \mathrm{UV}$ light in an Uvitec CL508S UV Crosslinker. Cultures were subsequently centrifuged again at $4000 \mathrm{rpm}$ in a swing bucket centrifuge (Sigma, 4K15 ) for 20 mins. Pellets were resuspended in lysis buffer $(20 \mathrm{mM}$ Tris $\mathrm{pH} 8.0,1 \mathrm{mM} \mathrm{MgCl}$, $150 \mathrm{mM} \mathrm{KCl}$, supplemented with 1 Complete protease inhibitor tab (Roche, 11697498001), 1 phosphoSTOP phosphatase inhibitor tab (Roche, 04906837001) and $0.2 \mathrm{U} / \mathrm{ml}$ RNasin (Promega, N251B per $30 \mathrm{ml}$ ) and lysed by 7 cycles of mechanical agitation in with Lysing Matrix B glass beads (MP Biomedicals, 6911-100) in a FastPrep apparatus (speed 6.5, 45 secs followed by $1 \mathrm{~min}$ on ice). Lysates were centrifuged in a tabletop centrifuge for 2 mins at $13,000 \mathrm{rpm}$ to clarify lysates. $100 \mu \mathrm{l}$ of lysate was removed and used for total RNA isolation. The remaining lysate was added to $40 \mu$ l of $\alpha$-FLAG M2 affinity gel (Sigma, A2220) pre-equlibrated with two $200 \mu \mathrm{l}$ washes in lysis buffer and incubated at $4^{\circ} \mathrm{C}$ for $4 \mathrm{hrs}$ with agitation. Beads were subsequently washed 4 times for 15 mins in lysis buffer followed by elution with three washes of $80 \mu \mathrm{l}$ $200 \mu \mathrm{g} / \mathrm{ml}$ 3X FLAG peptide (Sigma, F4799). RNA was subsequently isolated as described above with Tri Reagent (Sigma, 93289) according to the manufacturer's instructions, with the addition of glycogen (Ambion, AM9510) to the precipitation step to a concentration of $0.05 \mu \mathrm{g} / \mu \mathrm{l}$.

\section{RNA-Seq}

All column purifications were performed with RNA Clean \& Concentrator-5, Zymo Research (Proteigene, R1015) following manufacturer's recommendations. 400-600 ng of RNA was fragmented 5 mins (Ambion, AM8740) and purified. RNA was subsequently treated with $2 U$ of Antartic Phosphatase (NEB, M0289S) for 30 mins at $37^{\circ} \mathrm{C}$ and the enzyme was heat inactivated by heating for 5 mins at $65^{\circ} \mathrm{C}$. RNA fragments were $5^{\prime}$ monophosphorylated with $20 \mathrm{U}$ of T4 polynucleotide kinase (NEB, M0201S) and $1.667 \mathrm{mM}$ ATP for 1 hour at $37^{\circ} \mathrm{C}$ before a column purification. Libraries were constructed using NEBNext Multiplex Small RNA Library Prep Set for Illumina (Set 1) (NEB, E7300S), adjusting adapter concentrations as suggested in manufacturer's recommendations. Library were subsequently purified with AMPure XP beads (Agencourt, A63880) and checked for quality with Bioanalyzer DNA High Sensitivity Chips (Agilent, 5065-4626). Sequencing was performed on a MiSeq (Illumina) in a multiplexed single read using a MiSeq Reagent Kit v3 (Illumina, MS-102-3001). Raw sequence files (fastq) were cleaned of $3^{\prime}$ Adapter sequences (AGATCGGAAGAGCACACGTCTGAACTCCAGTCACNNNNNNATCTCGTATG CCGTCTTCTGCTTG), and low quality (cutoff 28) and short sequence (15nt) reads were removed using AdapterRemoval v1.5.2 (Lindgreen et. al, 2012) 
software. Reads were aligned against the reference genome (NC_003210) with bowtie v1.0.0 (Langmead et al., 2009) using default parameters, counted with HTSeq v0.6.0 and normalized to reads per million.

\section{SDS-PAGE and Western blot}

Samples were denatured at $95^{\circ} \mathrm{C}$ for 5 minutes and separated on a $12 \%$ SDS-PAGE before being transferred with a iBlot apparatus (Invitrogen, IB1001) using the $\mathrm{P} 6$ protocol for 6 mins according to the manufacturer's instructions. The membrane was blocked in 5\% powder milk in TBS-Tween for two hours at room temperature. Primary antibody, a-FLAG (Sigma, F7425) (dilution 1:1000) was incubated overnight at $4^{\circ} \mathrm{C}$. Membranes were washed $4 \times 10$ minutes in TBSTween at room temperature. Secondary antibody, a-rabbit-HRP (Agrisera) was incubated for 1 hour at RT (1:10000 dilution) in TBS-Tween before washing the membrane as described above. The membrane was developed using ECL2 prime Western Blot Detection (Pierce, 11884584) according to the manufacturer's instructions and subsequently exposed (LAS 4000, Fujifilm). 
Figure S1

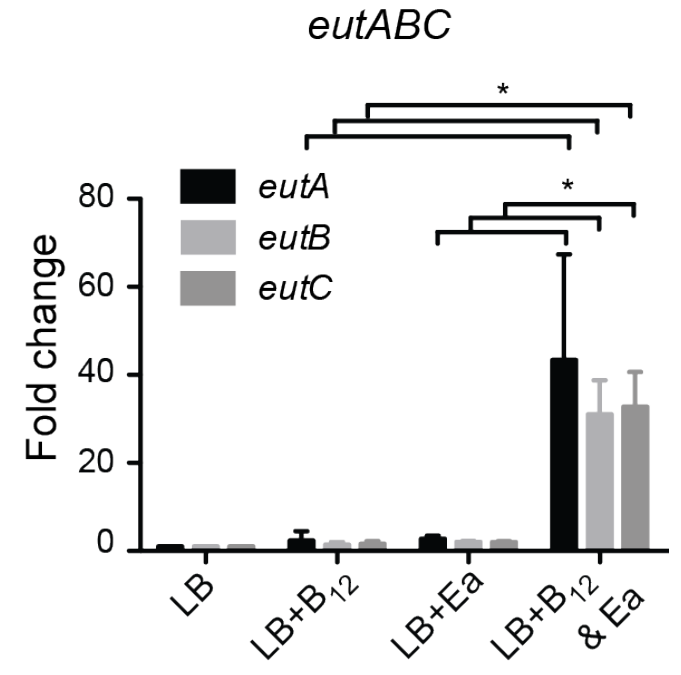

eut $A B C$ genes are regulated by both $B_{12}$ and ethanolamine. Expression levels of the eutABC genes were evaluated by qRT-PCR. RNA was isolated from bacteria grown in $\mathrm{LB}, \mathrm{LB}+20 \mathrm{nM} \mathrm{B} \mathrm{B}_{12}\left(\mathrm{LB}+\mathrm{B}_{12}\right)$, LB plus $10 \mu \mathrm{M}$ ethanolamine $(\mathrm{LB}+\mathrm{Ea})$ or LB plus or LB + both $\left(L B+B_{12} \& E a\right)$ to an $O D_{600}$ between 0.3 and 0.5 . Results represent the average of three biological replicates. Values depict means $+/-\mathrm{SEM},{ }^{*}=p<0.05$ 
Figure S2

A
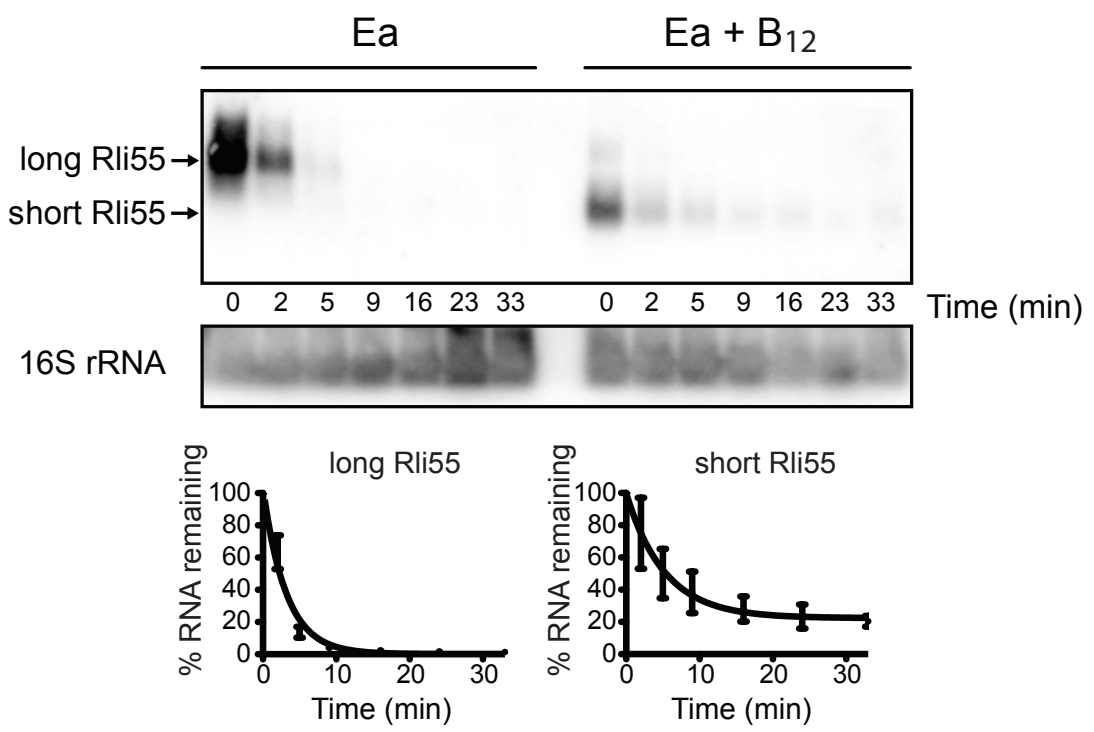

B

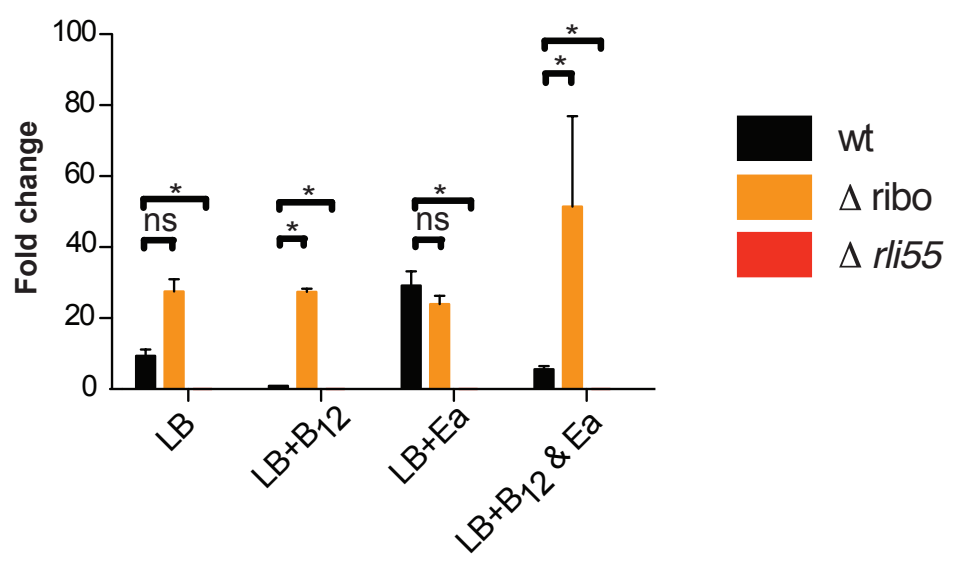

Rli55 stability and regulation. (A) northern blot showing expression of long and short Rli55 transcripts. RNA was isolated at the indicated timepoints after addition of Rifampicin and Naladixic acid to cultures. Blots were probed with a radioactively labeled, single-stranded RNA probe complimentary to the $B_{12}$ riboswitch. Northern blotting of $16 \mathrm{~S}$ rRNA is shown as a loading control. Graphs show decay of RNA overtime. Value represent means +/- SD, $n=3$ (B) Expression of rli55 was evaluated by qRT-PCR. RNA was isolated from bacteria grown in $L B, L B+20 n M B_{12}\left(L B+B_{12}\right), L B+10 \mu M$ ethanolamine $(\mathrm{LB}+\mathrm{Ea})$ or both $\left(\mathrm{LB}+\mathrm{B}_{12}\right.$ \& Ea) to an $\mathrm{OD}_{600}$ between 0.3 and 0.5 . Results represent the average of three biological replicates. Error bars show standard error. Values represent means $+/-\operatorname{SEM}, n=3,{ }^{*}=p<0.05, n s=$ not significant. 
Figure S3

A

eutA

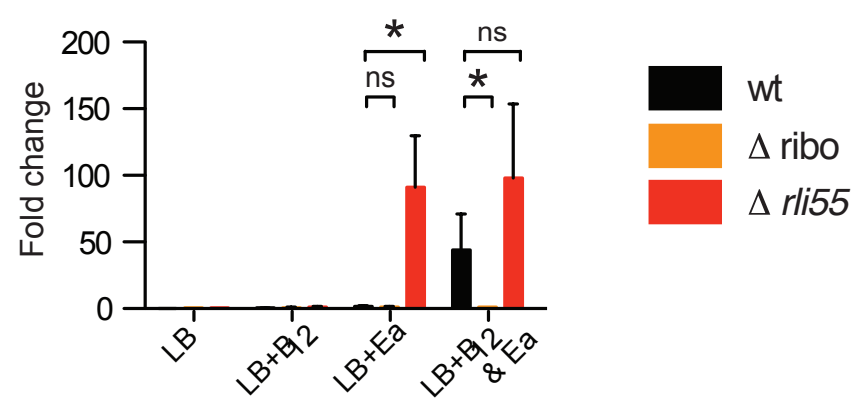

B

eutB

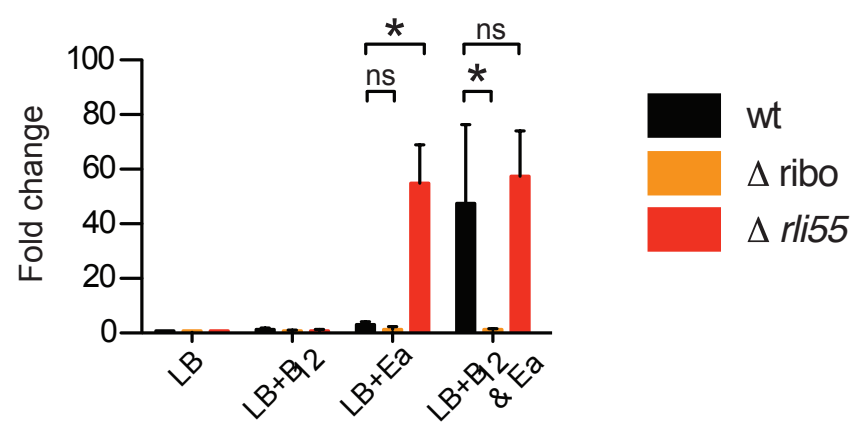

C

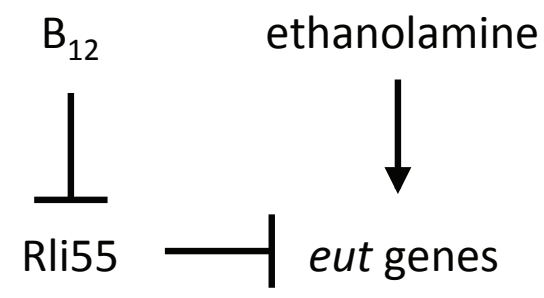

Rli55 regulates eut gene expression. Levels of the (A) eutB \& (B) eutC genes were evaluated by qRT-PCR in the wild-type (wt), $\Delta$ ribo or $\triangle$ rli55 strains. RNA was isolated from bacteria grown in LB, LB + $20 \mathrm{nM} B_{12}\left(L B+B_{12}\right)$, LB $+10 \mu \mathrm{M}$ ethanolamine (LB + Ea) or both ( $\left(\mathrm{BB}+\mathrm{B}_{12} \& \mathrm{Ea}\right)$ to an $\mathrm{OD}_{600}$ between 0.3 and 0.5 . Results represent the average of three biological replicates and error bars show standard error. Values represent means +/- SEM, Asterisks denote statistically significant differences $(p<0.05)$, ns $=$ not significant. (D) Model of the regulatory interactions between $B_{12}$, ethanolamine, Rli55 and eut gene expression. 
Figure S4

A

E. faecalis V583
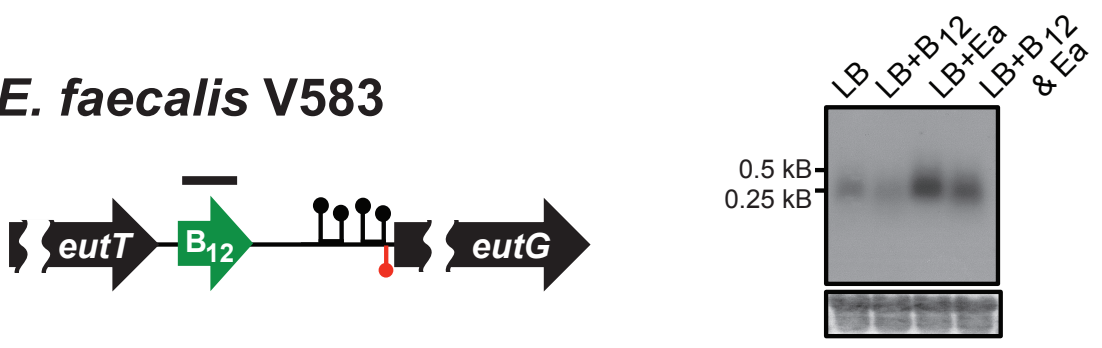

B

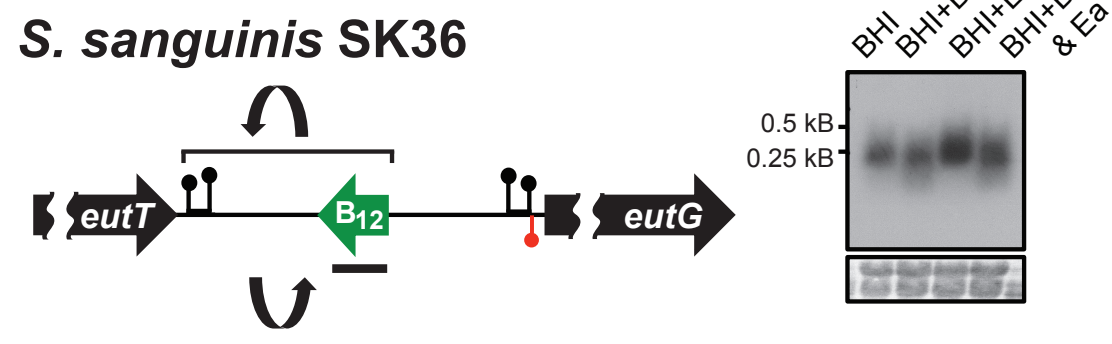

$$
\begin{aligned}
\text { ฯ } & =\text { ANTAR element } \\
\downarrow & =\text { Terminator }
\end{aligned}
$$

Putative Rli55 Orthologues. Schematics show the orthologous rli55 loci of E. faecalis and $S$. sanguinis, including $B_{12}$ riboswitches (green arrows), ANTAR elements (black double lollipops) and terminators (red inverted lollipops). An inversion of the $B_{12}$ riboswitch and a single ANTAR element in $S$. sanguinis (area within brackets) is denoted by arced arrows. Northern blots show transcripts detected with single stranded RNA probes directed against the $B_{12}$ riboswitch from each locus (black solid bars) using RNA isolated from bacteria grown in either $\mathrm{LB}$ or $\mathrm{BHI},+20 \mathrm{nM} \mathrm{B}_{12}\left(+\mathrm{B}_{12}\right), 10 \mu \mathrm{M}$ ethanolamine $(+\mathrm{Ea})$ or both $\left(+\mathrm{B}_{12} \& \mathrm{Ea}\right)$ as indicated. 
Figure S5

A

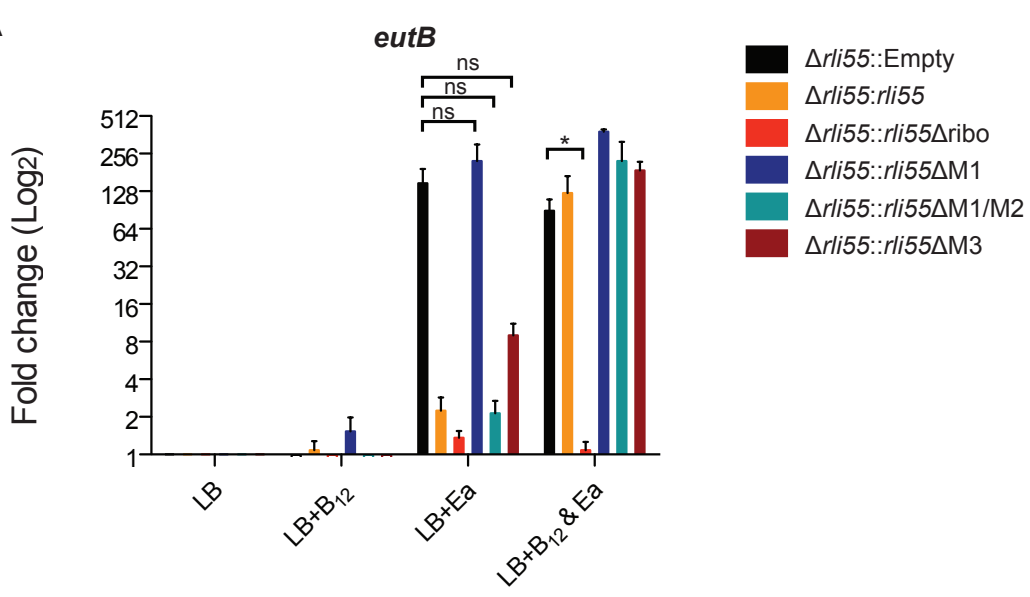

B

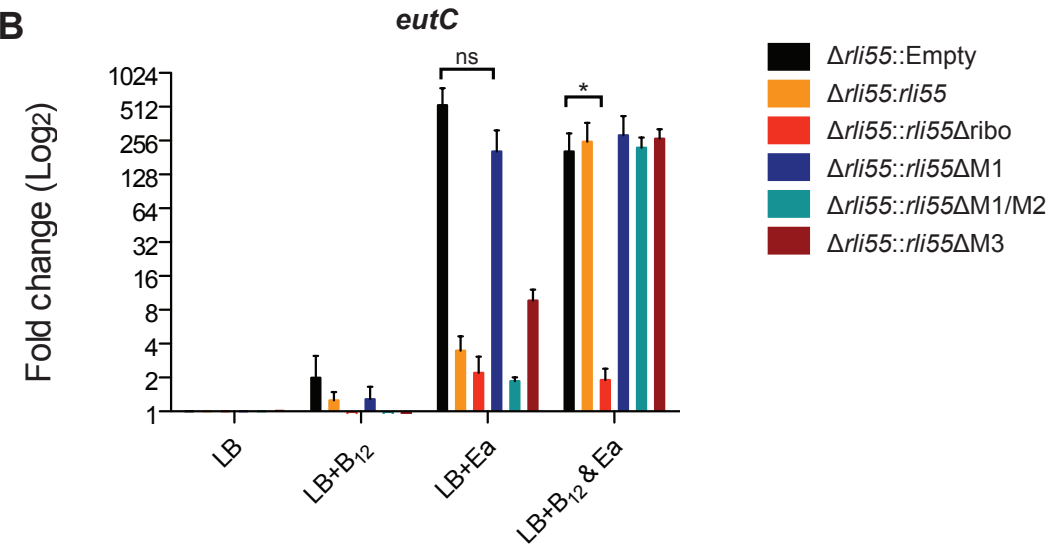

C

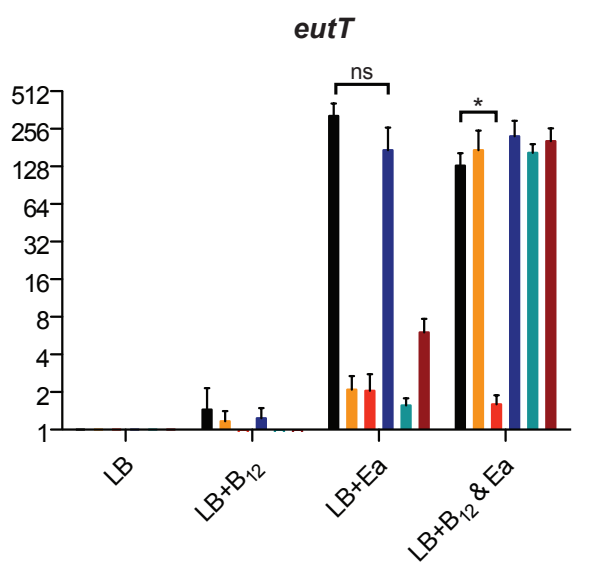

$\Delta$ rli55::Empty

$\Delta$ rli55:rli55

$\Delta$ rli55::rli55 4 ribo

$\Delta r l i 55:: r l i 55 \Delta \mathrm{M} 1$

$\Delta r l i 55:: r l i 55 \Delta \mathrm{M} 1 / \mathrm{M} 2$

$\Delta r l i 55:: r l i 55 \Delta \mathrm{M} 3$

Complementation of Rli55-mediated regulation. Expression of the (A) eutB, (B) eutC \& (C) eutT genes was evaluated by qRT-PCR. Graphs show the fold-change relative to the reference condition (LB) in $\log _{2}$ units for the indicated strains grown in LB, $L B+20$ $n M B_{12}\left(L B+B_{12}\right), L B+10 \mu M$ ethanolamine $(L B+E a)$ or both $\left(L B+B_{12} \& E a\right)$. Values represent means $+/$ - SEM, $n=3$. Asterisks denote statistically significant differences $(p<0.05)$, ns=not significant. 
Figure S6

A

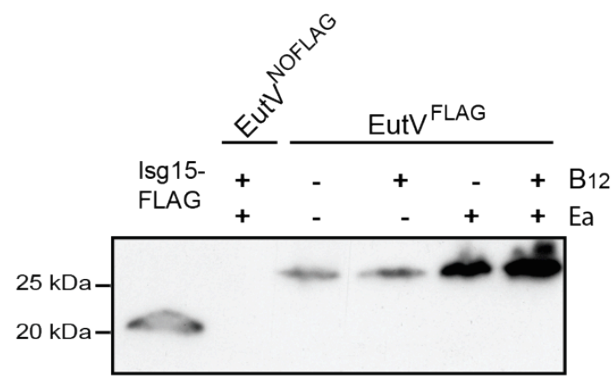

B

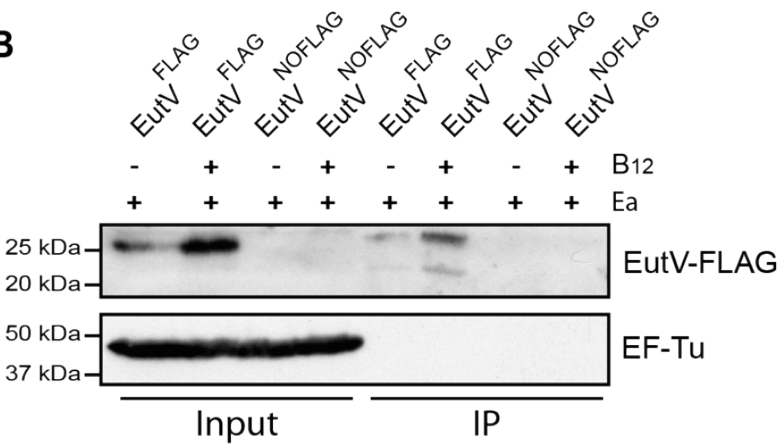

C

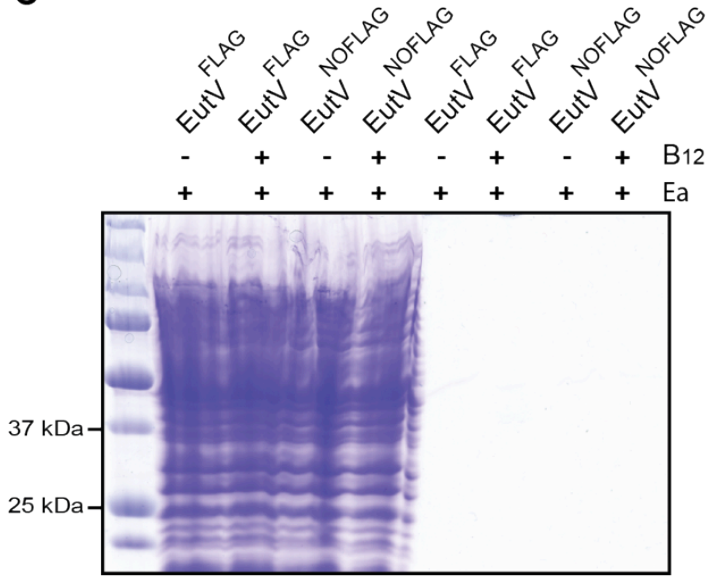

Regulation and immunoprecipitation of $\operatorname{EutV}^{F L A G}(A)$ Western blot showing regulation of EutV ${ }^{\text {FLAG }}$ under the indicated growth conditions. A purified human Isg $15^{\text {FLAG }}$ protein and a lysate of strain EutV ${ }^{\text {NOFLAG }}$, carrying and additional untagged eut $V$ gene grown in the presence of both $B_{12}$ and ethanolamine were included as controls. (B) Western blots of protein isolated from lysates of EutV ${ }^{F L A G}$ and EutV ${ }^{N O F L A G}$ strains grown either in ethanolamine (Ea) alone of ethanolamine and $B_{12}$ together either before (input) or after immunoprecipitations (IP) with $\alpha$-FLAG affinity gel. Blots of the EF-Tu protein were evaluated as a control. (C) A Commassie stained gel run in parallel shows the overall protein content of all lanes. 


\section{Figure $\mathbf{S 7}$}

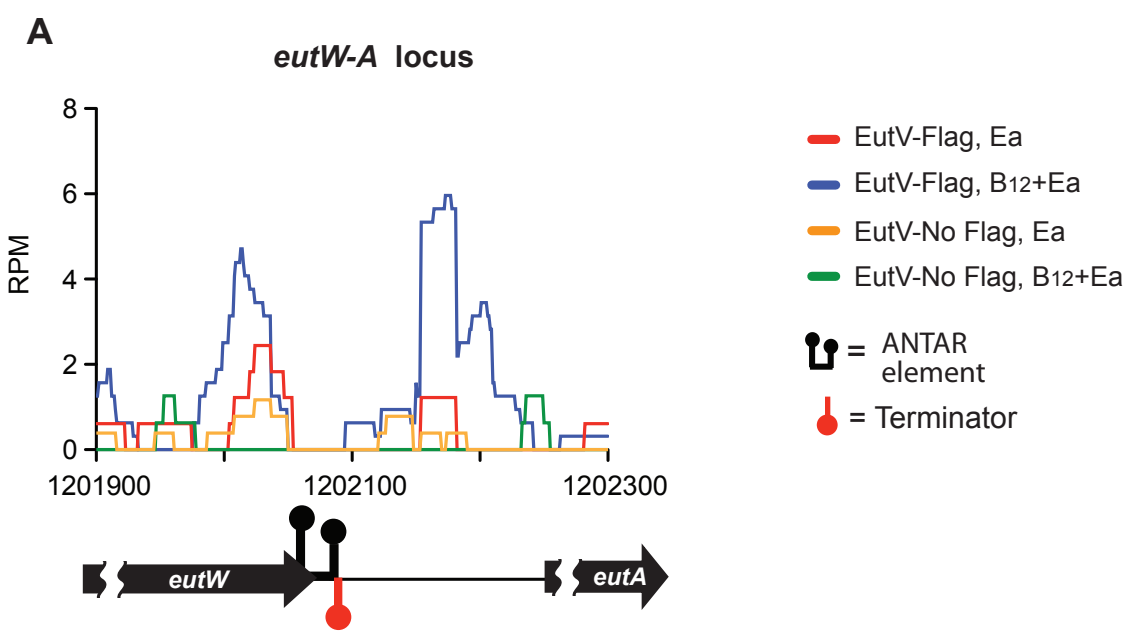

B

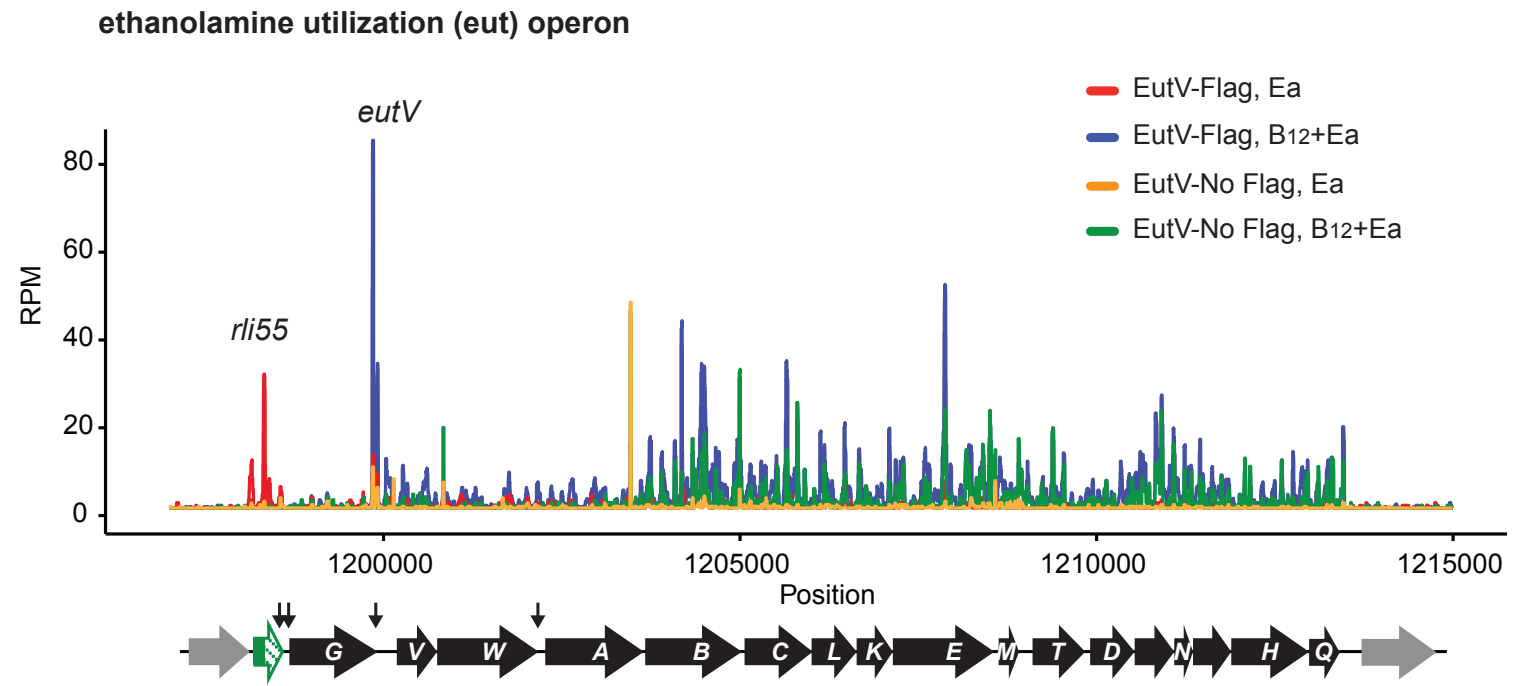

Enrichment of the eut operon by colP with EutV ${ }^{\text {FLAG }}$. Histograms shows RNA-Seq coverage in reads per million (RPM) of the (A) eutW - eutA intergenic region and the (B) full eut locus with RNA isolated by co-immunoprecipitation of cell lysates from either EutV ${ }^{\text {FLAG }}$ or EutV ${ }^{\text {NOFLAG }}$ cultures grown in the presence of ethanolamine (Ea) or ethanolamine and $B_{12}$ together. Schematics below show each loci. Arrows in the second schematic indicate positions of ANTAR elements. 


\section{Table S1}

Strains and plasmids used in this study

\begin{tabular}{|c|}
\hline $\begin{array}{l}\text { Strains } \\
\text { Listeria monocytogenes } \\
\text { EGDe (BUG1600) }\end{array}$ \\
\hline$\Delta$ ribo (BUG3593) \\
\hline Arli55 (BUG3594) \\
\hline Arli55::Empty (BUG3550) \\
\hline Arli55::rli55 (BUG3551) \\
\hline$\Delta$ rli55::Aribo (BUG3552) \\
\hline$\Delta$ rli55::rli55 $\mathrm{M} 1$ (BUG3553) \\
\hline$\Delta r l i 55:: r l i 55 \Delta \mathrm{M} 1 / \mathrm{M} 2$ (BUG3554) \\
\hline 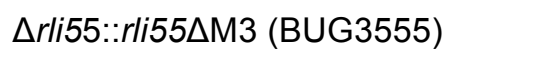 \\
\hline EutV $^{\text {rLAG }}$ (BUG3559) \\
\hline EutV $V^{\text {NUrLAG }}$ (BUG3558) \\
\hline
\end{tabular}

Streptococcus sanguinis SK36

Enterococcus faecalis V583

E. coli Top 10

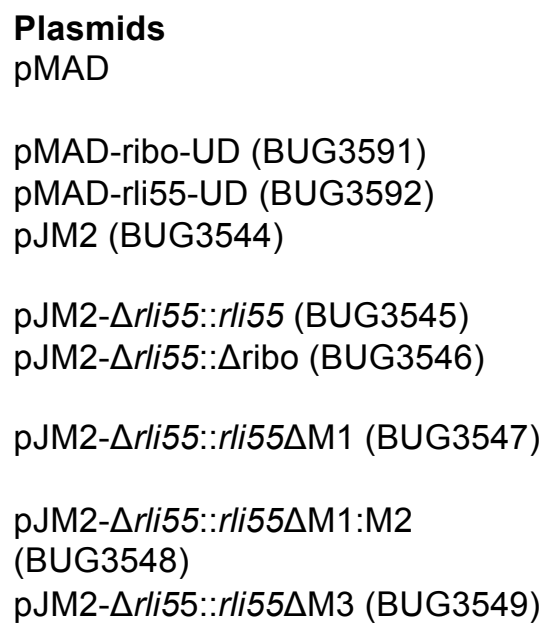

Plasmids pMAD

pMAD-ribo-UD (BUG3591)

pMAD-rli55-UD (BUG3592) pJM2 (BUG3544)

pJM2- $\Delta$ rli55::rli55 (BUG3545)

pJM2- $\Delta$ rli55:: $\Delta$ ribo (BUG3546)

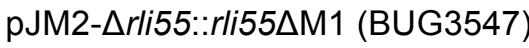

pJM2-Arli55::rli55 $\Delta \mathrm{M} 1: \mathrm{M} 2$

(BUG3548)

pJM2-Arli55::rli55 $\Delta$ M3 (BUG3549)

Mutant containing a deletion of nucleotides 1198141-1198190 encompassing a portion of the $\mathrm{B} 12$ riboswitch at this position.

Mutant containing a deletion of nucleotides 1198066-1198566 encompassing the B12 riboswitch and downstream region.

Strains with named plasmids integrated into the tRNA ${ }^{\text {ARG }}$ locus

Strains with named plasmids integrated into the tRNA ${ }^{A R G}$ locus

Strains with named plasmids integrated into the tRNA ${ }^{A R G}$ locus

Strains with named plasmids integrated into This study the tRNA ${ }^{A R G}$ locus

Strains with named plasmids integrated into This study the tRNA ${ }^{\mathrm{ARG}}$ locus

Strains with named plasmids integrated into the tRNA ${ }^{A R G}$ locus

Strrain carrying a $2 X$ FLAG-tagged additional copy of the eutV gene in the tRNAARG locus

Strrain carrying an untagged additional copy of the eutV gene in the tRNAARG locus

Mackaness

GB et al, 1964

This study

This study

This study

This study

This study

This study

This study

This study

Xu P et al., 2007

F- mcrA $\Delta$ (mrr-hsdRMS-mcrBC) $\varphi 80$ lacZ $\Delta M 15$ $\Delta$ lacX74 recA1 araD139 $\Delta$ (ara-leu) 7697 galU

Invitrogen, C4040 galK rpsL (StrR) endA1 nupG $\lambda$ -

shuttle vector used for creating plasmids for mutagenesis

plasmid used to create the $\Delta$ ribo mutant plasmid used to create the $\triangle$ ribosRNA mutant derived from $\mathrm{pAD}$ vector (Balestrino et al. 2010)

pJM2 vector carrying wild-type rli55 allelle

pJM2 vector carrying rli55 allelle with

$\triangle$ mutation

pJM2 vector carrying rli55 allelle with $\Delta \mathrm{M} 1$

ANTAR mutation

pJM2 vector carrying rli55 allelle with $\Delta \mathrm{M} 1$ and

$\triangle \mathrm{M} 2$ ANTAR mutations

pJM2 vector carrying rli55 allelle with $\triangle M 3 \quad$ This study
Arnaud $\mathrm{M}$ et al. 2004

This study

This study

This study

This study This study

This study

This study 
Table S2

Name

Sequence

Oligonucleotides used to create mutants

$\Delta$ rli55-F

GGTCTTTTATCTAATTGTTTTAACTG

$\Delta$ rli55-R

CAGTTAAAACAATTAGATAAAAGACCACCAGACTTCCAGTATCTAAC $\mathrm{T}$

$\Delta$ ribo-Up-F

AATGACCCGGGTCCGAAGTCACTTTATCCGATAAA

$\Delta$ ribo-Up-R

AGAGAAATTTTCTCTTCCAACTCATTGCAAGAAGAATTTGCTTTACG

AA

$\Delta$ ribo-Down-F

TGAGTTGGAAGAGAAAATTTCTCT

$\Delta$ ribo-Down-R

TCGAG GTCGAC TCTTTGTAAGCACGTTCTAAGTAT

$\Delta$ eutB-Up-F

AATGACCCGGGTGAATGTATCTCTTTTTCCGGAG

$\Delta$ eutB-Up-R

$\Delta$ eutB-Down-F

$\Delta$ eutB-Down-R

TCGCTTAGTTCCTCCCTTCCAGCTATTTATGTGGATCAGTTAAAG

CTTTAACTGATCCACATAAATAGCTGGAAGGGAGGAACTAAGCGA

TCGAGGTCGACTCACCTACAAGTAAGCAAACTAC

Oligonucleotides used to create T7 templates for Northern blot probes

T7riboprobetemp-F

TTCATACAAACAGTTAGATACTG

T7riboprobetemp-F

ACGTTAATACGACTCACTATAGGGAGAATAAAACGAAGCGGTGCAC

T7rli55downstream-F

T7rli55downstream-R

A

TGCACCGCTTCGTTTTATGGT

ACGTTAATACGACTCACTATAGGGAGAAGCTTTGAGTAGAGAAATTT

TCTCT

T7Efriboprobetemp-F

T7Efriboprobetemp-R

ACGTTAATACGACTCACTATAGGGAGA GAAACATCCTGTTTTGCC

CGAATATTTTATGAAAAATATGAATGGAA

T7Ssriboprobetemp-F

ACGTTAATACGACTCACTATAGGGAGAATTTAAGAATAAAGACCTGA

CTTAA

T716S-F

ATGCCACGCCTTTGAAAGATG

T716S-R

ACGTTAATACGACTCACTATAGGGAGATGCACTCCAGTCTTCCAGT

TT

T7Ssriboprobetemp-R AATTGATTTTAGTACTTTTAAGGATA

Oligonucleotides used for qRT-PCR

qRTrli55-F

qRTrli55-R

qRTeutA-F

qRTeutA-R

qRTeutB-F

qRTeutB-R

qRTeutC-F

qRTeutC-R

qRTeutT-F

qRTeutT-R

qRTeutV-F

qRTeutV-R

qRTrpoB-F

qRTrpoB-R

ACAAATAAGCCCACTGTAAATG

AAACCAAACACGTGGAAATG

ATCTCAACGGGTGCGCTAAGTGAT

AATTTCAGCTGTGTGTGAACCGGC

ACTGGTTACCACGAAACAGCCACT

ATGCATCTCCAGCACGGCTAGTTA

AAATCGGTGAAATCACTGGCGCTG

AACTGTACGACGAGCTTCTGGCAT

GACGCATTTGCGTGGTAATCTGCT

TGCAACGGAACATTGGGTTTCCAG

TTATTTCAGAAGGGCTTGCTGGCG

GTTTCTCGCCCTTTGGCAATACTC

GCGAACATGCAACGTCAAGCAGTA

ATGTTTGGCAGTTACAGCAGCACC

Oligonucleotides used for $\Delta$ rli55 complementation constructs

rli55-F

rli55-R

AATGACCCGGGAGCAAATTCTTCTTGCAAGCGG

rli55 $\triangle \mathrm{M} 1-\mathrm{F}$

rli55 $\triangle \mathrm{M} 1-\mathrm{R}$

rli55 $\Delta \mathrm{M} 1: \mathrm{M} 2-\mathrm{F}$

rli55 $\triangle \mathrm{M} 1: \mathrm{M} 2-\mathrm{R}$

rli55 $\triangle \mathrm{M} 3-\mathrm{F}$

rli55 $\Delta \mathrm{M} 3-\mathrm{R}$

AATGAGTCGACGAGATTACCTCCTAGTTTTTTTAAAATA

GGTGGGCAATGATCAAAACAACGACGAAAAGA

TCTTTTCGTCGTTGTTTTGATCATTGCCCACC

TCAAAACAACGACGTTTTGAGTGATACAAGG

CCTTGTATCACTCAAAACGTCGTTGTTTTGA

AAATAAAATATTTTAGTCATGAAAGGCGTACTGTTTACTT

AAGTAAACAGTACGCCTTTCATGACTAAAATATTTTATTT 